\title{
Small-Scale Farmer Preferences: Exploring the Gap Towards Product Attributes of Local and Imported Fertilizers
}

\author{
Tomy Perdana ${ }^{\#}$, Hesty Nurul Utami", Agriani Hermita Sadeli", Fernianda Rahayu Hermiatin \\ \# Department of Agricultural Economics and Social Sciences, Universitas Padjadjaran, Jatinangor, 45363, Indonesia \\ E-mail: tomy.perdana@unpad.ac.id, hesty.nurul@unpad.ac.id, agriani.hermita@unpad.ac.id
}

*Agricultural Logistics and Supply Chain System (AGRILOGICS) Research Group, Universitas Padjadjaran, Jatinangor, 45363, Indonesia

E-mail: ferniandarahayu@gmail.com

\begin{abstract}
Marketing program becomes a crucial matter for fertilizer industries to boost selling in a particular marketplace. Local fertilizer industries actors are tried to outperform the competitor from other countries. The research objective is to explore horticulture small-scale farmer preference for product attributes of local and imported fertilizer. The research was conducted in Java, Indonesia as a representative region of horticultural farmers. This study used a survey method to gather data from 513 smallscale farmers horticultural farmers which spread over four provinces in Java Island. The data were analyzed with MANOVA analysis. The result shows that farmers have a similar preference for local and imported fertilizers concerning price, promotion, and nutrient content. Farmers prefer price between IDR 16,000 to IDR 20,000 per $\mathrm{kg}$, promotion through farm demonstration plot, and product content additional micro-nutrients and vitamins. On the other hand, there is a distinct preference for the design of fertilizer packaging. Farmers prefer imported fertilizer with an additional image, but not on local fertilizers. This finding serves as a reference to fertilizer producers in improving their marketing strategy. Local fertilizers producers should undertake a strategy adjusted to farmer preferences to win the competition in local market. This research only represents the behavior on the small-scale farmers, the result might be different if it apply in a large-scale farmer because this type of farmer has different characteristic which will influence to their preference of fertilizer.
\end{abstract}

Keywords - customer preference; marketing strategy; fertilizer; small-scale farmer.

\section{INTRODUCTION}

Today the transformation of agricultural system in developing countries is greatly fast. Due to the development of agricultural system, farmers become selective to buy agricultural-input products, e.g. seeds, fertilizers, pesticides and supporting cultivation machinery [1]. Besides that, consumers use various information processing strategies to consider products and to reach their decisions [2]. Product attribute determination process will bring the successful prediction of customer purchase [3]. Producer countries and product quality of available product categories significantly affect consumer decision making [4].

In Indonesia, small-scale farmers as consumers fulfill their need from local and imported product. Considering that fertilizer becomes compete for the product in the global industry. Indonesian fertilizer producers compete with other countries producers as strong competitors in order to win the market. Therefore, effective marketing strategies are one of crucial functions in fertilizer organization management [5].
It is important to explore small-scale horticultural farmers preferences for product attribute of local and imported fertilizers in Java Island, Indonesia as a production center of the horticultural commodity in Indonesia.

Fertilizers are an essential element in the cultivation activities to improve plant productivity. Farming sustainability can be carried out by maintaining or increasing soil [5]. Most farmers use fertilizers in farming activities as nutrient supplement for soil and plants [7]. Because of the importance of fertilizer, farmer must choose the appropriate fertilizer to improve their farming result.

There are different evaluations of imported products with similar domestic products [8]. Globalization and free trade in the economic situation give a high impact and all at once hamper for local fertilizer industries. In line with that condition, fertilizer consumers in developing countries have many preferences to decide in purchasing fertilizer. Consumer preferences depend on the subjective perspective and taste as measured by the utility of various goods [9].

The underlying foundation of fertilizer demand is a model of how consumer have. The consumer's wants are unlimited 
and outpace customer ability to satisfy them all. Consumer preference gives a positive influence on product consumption behaviour [10]. Recently, farmers as fertilizer customers prefer to purchase a better quality of fertilizer rather than to consider the price [11]. Moreover, farmers become selective to use fertilizer while consideringof nutrient content and primary ingredient of fertilizer [12]. Price, brand status, product labelling, and nutrient determine customer purchasing preferences for fertilizer [13]. Brand and packaging are representative of product quality that creates customer value [14]. Product labelling as a packaging elements is an important part because some consumer perceive packaging as a visual aestechics [15]. The emerging farmers' fertilizer purchase decision is strongly influenced by service, brand, product, and learning or psychological factors [5]. One way to learn about the fertilizer is through demonstration plot, as a promotional medium from the fertilizer company.

According to Statistics in Indonesia in the last eleven years of 2007 - 2017, the average consumption of imported fertilizers in Indonesia was over $40.51 \%$. On the other hand, the market size of fertilizer in 2017 was up to 3.1 million tons or rose $6.24 \%$ from 2016 [16]. It is described that fertilizer consumption tends to increase year by year considering several factors (such as climate change, type of farmland, nutrient needs per plant, etc.). The use of fertilizer in Asia dramatically increases due to the environment condition; fertilizer is considered capable of controlling weeds, pests and diseases, and optimum utilization of soil moisture from rainfall and irrigation. Fertilizer subsidies regarding the government policies have taken a roll in the fertilizer marketplace [17]. These, successively, have spurred a re-evaluation of fertilizer companies, in particular local fertilizer companies to escalate the competitiveness in the marketplace.

There are rare researches about customer preference for fertilizers. The previous research discuss fertilizer as a general without consider the location of fertilizer producer in terms of geographical area such as producer from local and other countries. In addition, there are no research discuss about the behavior of farmer fertilizer preference in terms of business size, to be more specific in small holders. Therefore, it is important to conduct a study about small-scale farmer preferences for the local and imported fertilizers in Indonesia. In this research, consumer preference for fertilizer product attribute is examined by price, promotion, nutrient content and design of the packaging. This research gives an overview about farmer behavior in particular their preference for fertilizer. It may used as reference for fertilizer producer to decide marketing strategy in particular attribute product. In addition, it contribute to the agricultural marketing knowledge to understand the behavior of farmer specifically in small-scale farmer.

\section{MATERIAL AND METHOD}

This research is based on horticultural farmer's preference for using fertilizers at a horticulture production center in Java Island of Indonesia covering West Java province, Central Java Province, East Java province and Banten Province. The target sample of the survey was selected using cluster sampling technique. The sample size in this study was 513 respondents which spread over four provinces in Java Island who were small-scale horticultural farmers that used local and imported fertilizers frequently. Data were collected through survey method using a questionnaire with multiple choice questions. The questions were about the customer preferences for fertilizer attribute product in terms of price [11], [13] promotion through demonstration plot where farmers could learn [5], nutrient [5], [12], [13], and design of packaging [15]. Price attribute was examined by the amount of money that farmers were willing to pay for the product. Promotion was determined by the type of promotion preferred by the consumer to ensure trust and the knowledge of the product such as land for demonstration, farmer training, farmer assistance, and free sample products. Nutrient content is the material contained in the fertilizer. Design of packaging was examined by design of packaging that farmers preferred.

In this research, customer preference is the dependent variable in quantitative data. Customer preference is divided into two categories namely customer preference for local and imported fertilizers. MANOVA analysis test examines the significance of the mean of two different groups [18]. MANOVA test procedures are as follows:

\section{A. The two different groups are significantly different from several independent variables.}

Significance test shows centroid difference between the two groups with multivariate significance test. Hypotheses are as follows:

$$
H_{0}:\left(\begin{array}{l}
\mu_{1_{1}} \\
\mu_{2_{1}} \\
\mu_{3_{1}} \\
\mu_{4_{1}}
\end{array}\right)=\left(\begin{array}{l}
\mu_{1_{2}} \\
\mu_{2_{2}} \\
\mu_{3_{2}} \\
\mu_{4_{2}}
\end{array}\right) \quad H_{1}:\left(\begin{array}{l}
\mu_{1_{1}} \\
\mu_{2_{1}} \\
\mu_{3_{1}} \\
\mu_{4_{1}}
\end{array}\right) \neq\left(\begin{array}{l}
\mu_{1_{2}} \\
\mu_{2_{2}} \\
\mu_{3_{2}} \\
\mu_{4_{2}}
\end{array}\right)
$$

\section{B. Multivariate Significance Test}

The test is to know the difference of two groups' centroid that can be evaluated with (1) Pillai's Trace; (2) Hotelling's Trace; (3) Wilks' Lambda; and (4) Roy's Largest Root. Afterward, the forth-multivariate statistics test is transformed into F-ratio test.

$\begin{array}{ll}\text { Pillai's Trace } & \sum_{i}^{k}=1 \frac{\lambda_{i}}{1+\lambda_{i}} \\ \text { Hotelling's Trace } & \sum_{i}^{k}=1\left(\lambda_{i}\right) \\ \text { Wilks Lambda } & \prod_{i}^{k}=1 \frac{1}{1+\lambda_{i}} \\ \text { Roy's Largest Root } & \frac{\lambda_{\max }}{1+\lambda_{\max }}\end{array}$

\section{Effect Size}

Effect size is used to determine whether any significance mean or group of centroid with Partial Eta Squares (PES) has differences in size.

$$
\mathrm{PES}=1-\Lambda=1-\frac{S S_{b}}{S S_{w}}=\frac{S S_{b}}{S S_{t}}
$$

where $\mathrm{SS}_{\mathrm{b}}=$ sum of squares between group, $\mathrm{SS}_{\mathrm{w}}=$ sum of squares within group and $\mathrm{SS}_{\mathrm{t}}=$ sum of squares total. 


\section{Interaction test}

As the study has three independent variables, it means it has three main effects. With these three main effects, then it should run several tests. The first is the main effect test. The second is the main effect interaction test to recognize how each independent variable simultaneously influences the dependent variable.

\section{RESULTS AND DISCUSSION}

Farmers using fertilizers had different backgrounds in terms of age, sex, education and business governance as a farmer group. Table 1 shows the characteristics of farmer respondents. 76 percent of farmers were in the middle age. Almost all the farmers were male. Only 3.5 percent of farmers completed their bachelor and diploma degree and $92.8 \%$ had gone to elementary, junior and high school. Almost three quarter of farmers had no informal education. Less than 30 percent of farmers had an informal education in the form of training of cultivation, harvest and post harvest handling.

TABLE I

RESPONDENT CHARACTERISTICS

\begin{tabular}{|c|c|c|}
\hline Charcteristics & $\mathrm{n}$ & $\begin{array}{l}\text { Percentage } \\
(\%)\end{array}$ \\
\hline \multicolumn{3}{|l|}{ Age } \\
\hline Young Adult (20-29) & 19 & 3.7 \\
\hline Adult (30-39) & 68 & 13.3 \\
\hline Middle Age (40-60) & 390 & 76.0 \\
\hline Senior Age (More Than 61) & 36 & 7.0 \\
\hline \multicolumn{3}{|l|}{ Sex } \\
\hline Male & 501 & 97.7 \\
\hline Female & 12 & 2.3 \\
\hline \multicolumn{3}{|l|}{ Formal Education } \\
\hline None & 19 & 3.7 \\
\hline Elementary School & 243 & 47.4 \\
\hline Junior High School & 129 & 25.1 \\
\hline Senior High School & 104 & 20.3 \\
\hline Diploma & 6 & 1.2 \\
\hline Bachelor & 12 & 2.3 \\
\hline \multicolumn{3}{|l|}{ Informal Education } \\
\hline None & 370 & 72.1 \\
\hline Training & 143 & 27.9 \\
\hline
\end{tabular}

There are various types of fertilizer that are commonly used in Indonesia, such as Urea, NPK, ZA, phosphate/SP-36, $\mathrm{K} 2 \mathrm{SO} 4 / \mathrm{ZK}$ and organic fertilizer. Consumer preference is built by customer perspective of attribute products. Four attribute factors were analyzed as an indicator to measure consumer preference for fertilizer. Four attributes fertilizer used in this study are price, promotion, nutrient content and design of the packaging.

\section{A. Multivariate Normality Test}

The use of Box's test is to examine the multivariate normality test. The analysis showed significance result, which indicates the covariance matrix of dependent variables, where price, promotin, the design of packaging, and nutrient content are not the same. It explains that the multivariate normality assumption is not fulfilled. However, it should be taken into account that Box's $M$ test is very sensitive in terms of normality test violence. Multivariate analysis is robust; this means examination can be continued.

TABLE II

BOX's TEST OF EQUALITY OF COVARIANCE MATRICES

\begin{tabular}{|l|l|}
\hline Box's M & 187.684 \\
\hline F & 2.244 \\
\hline df1 & 69 \\
\hline df2 & 2486.895 \\
\hline Sig. & .000 \\
\hline
\end{tabular}

Bartlett's Test of Sphericity is used to show correlation test among the dependent variables. Bartlett's test shows a significant result that indicates the existence of correlation presents adequate evidence among observed independent variables, namely customer preference for local and imported fertilizers.

TABLE III

BARTLETT'S TEST OF SPHERICITY ${ }^{\mathrm{A}}$

\begin{tabular}{|l|l|}
\hline Likelihood Ratio & .000 \\
\hline Approx. Chi-Square & 830.968 \\
\hline df & 2 \\
\hline Sig. & .000 \\
\hline
\end{tabular}

\section{B. Multivariate Significance Test}

This study uses four types of statistical tests to know the difference between two groups of centroid, namely Pillai's Trade, Wilks Lambda, Hotelling's Trace and Roy's Largest Root. The results of these four statistic tests are transformed into F-test.

TABLE II

Multivariate Tests ${ }^{A}$

\begin{tabular}{|l|l|l|l|l|l|l|l|}
\hline \multicolumn{2}{|c|}{ Effect } & Value & F & Hypothesis df & $\begin{array}{c}\text { Error } \\
\text { df }\end{array}$ & $\begin{array}{c}\text { Sig. } \\
\text { Partial Eta } \\
\text { Squared }\end{array}$ \\
\hline \multirow{4}{*}{ Intercept } & Pillai's Trace & .744 & $671.703^{\mathrm{b}}$ & 2.000 & 463.000 & .000 & .744 \\
\cline { 2 - 8 } & Wilks' Lambda & .256 & $671.703^{\mathrm{b}}$ & 2.000 & 463.000 & .000 & .744 \\
\cline { 2 - 8 } & Hotelling's Trace & 2.902 & $671.703^{\mathrm{b}}$ & 2.000 & 463.000 & .000 & .744 \\
\cline { 2 - 8 } & Roy's Largest Root & 2.902 & $671.703^{\mathrm{b}}$ & 2.000 & 463.000 & .000 & .744 \\
\hline
\end{tabular}




\begin{tabular}{|c|c|c|c|c|c|c|c|}
\hline & Effect & Value & $\mathrm{F}$ & Hypothesis df & $\begin{array}{c}\text { Error } \\
\text { df }\end{array}$ & Sig. & $\begin{array}{l}\text { Partial Eta } \\
\text { Squared }\end{array}$ \\
\hline \multirow{4}{*}{ Price } & Pillai's Trace & .036 & 2.148 & 8.000 & 928.000 & .029 & .018 \\
\hline & Wilks' Lambda & .964 & $2.157^{\mathrm{b}}$ & 8.000 & 926.000 & .029 & .018 \\
\hline & Hotelling's Trace & .037 & 2.166 & 8.000 & 924.000 & .028 & .018 \\
\hline & Roy's Largest Root & .034 & $3.963^{\mathrm{c}}$ & 4.000 & 464.000 & .004 & .033 \\
\hline \multirow[t]{4}{*}{ Promotion } & Pillai's Trace & .056 & 4.428 & 6.000 & 928.000 & .000 & .028 \\
\hline & Wilks' Lambda & .945 & $4.468^{b}$ & 6.000 & 926.000 & .000 & .028 \\
\hline & Hotelling's Trace & .059 & 4.508 & 6.000 & 924.000 & .000 & .028 \\
\hline & Roy's Largest Root & .055 & $8.524^{\mathrm{c}}$ & 3.000 & 464.000 & .000 & .052 \\
\hline \multirow{4}{*}{$\begin{array}{l}\text { Nutrient } \\
\text { Content }\end{array}$} & Pillai's Trace & .061 & 4.861 & 6.000 & 928.000 & .000 & .030 \\
\hline & Wilks' Lambda & .940 & $4.861^{\mathrm{b}}$ & 6.000 & 926.000 & .000 & .031 \\
\hline & Hotelling's Trace & .063 & 4.861 & 6.000 & 924.000 & .000 & .031 \\
\hline & Roy's Largest Root & .043 & $6.706^{\mathrm{c}}$ & 3.000 & 464.000 & .000 & .042 \\
\hline \multirow{4}{*}{$\begin{array}{l}\text { Design of } \\
\text { Packaging }\end{array}$} & Pillai's Trace & .017 & $3.904^{\mathrm{b}}$ & 2.000 & 463.000 & .021 & .017 \\
\hline & Wilks' Lambda & .983 & $3.904^{\mathrm{b}}$ & 2.000 & 463.000 & .021 & .017 \\
\hline & \begin{tabular}{|l|} 
Hotelling's Trace \\
\end{tabular} & .017 & $3.904^{b}$ & 2.000 & 463.000 & .021 & .017 \\
\hline & Roy's Largest Root & .017 & $3.904^{\mathrm{b}}$ & 2.000 & 463.000 & .021 & .017 \\
\hline \multirow{4}{*}{$\begin{array}{l}\text { Price * } \\
\text { Promotion }\end{array}$} & \begin{tabular}{|l} 
Pillai's Trace \\
\end{tabular} & .016 & .742 & 10.000 & 928.000 & .685 & .008 \\
\hline & Wilks' Lambda & .984 & $.741^{\mathrm{b}}$ & 10.000 & 926.000 & .686 & .008 \\
\hline & Hotelling's Trace & .016 & .740 & 10.000 & 924.000 & .687 & .008 \\
\hline & Roy's Largest Root & .012 & $1.091^{\mathrm{c}}$ & 5.000 & 464.000 & .365 & .012 \\
\hline \multirow{4}{*}{$\begin{array}{l}\text { Price * Nutrient } \\
\text { Content }\end{array}$} & Pillai's Trace & .010 & .607 & 8.000 & 928.000 & .773 & .005 \\
\hline & Wilks' Lambda & .990 & $.606^{b}$ & 8.000 & 926.000 & .773 & .005 \\
\hline & \begin{tabular}{|l|} 
Hotelling's Trace \\
\end{tabular} & .010 & .605 & 8.000 & 924.000 & .774 & .005 \\
\hline & Roy's Largest Root & .008 & $.985^{\mathrm{c}}$ & 4.000 & 464.000 & .415 & .008 \\
\hline \multirow{4}{*}{$\begin{array}{l}\text { Price * } \\
\text { Design of } \\
\text { Packaging }\end{array}$} & \begin{tabular}{|l|} 
Pillai's Trace \\
\end{tabular} & .017 & 2.039 & 4.000 & 928.000 & .087 & .009 \\
\hline & \begin{tabular}{|l|} 
Wilks' Lambda \\
\end{tabular} & .983 & $2.041^{\mathrm{b}}$ & 4.000 & 926.000 & .087 & .009 \\
\hline & Hotelling's Trace & .018 & 2.043 & 4.000 & 924.000 & .086 & .009 \\
\hline & \begin{tabular}{|l} 
Roy's Largest Root \\
\end{tabular} & .016 & $3.736^{\mathrm{c}}$ & 2.000 & 464.000 & .025 & .016 \\
\hline \multirow{4}{*}{$\begin{array}{l}\text { Promotion * } \\
\text { Nutrient } \\
\text { Content }\end{array}$} & Pillai's Trace & .066 & 1.747 & 18.000 & 928.000 & .027 & .033 \\
\hline & Wilks' Lambda & .935 & $1.744^{\mathrm{b}}$ & 18.000 & 926.000 & .028 & .033 \\
\hline & Hotelling's Trace & .068 & 1.742 & 18.000 & 924.000 & .028 & .033 \\
\hline & Roy's Largest Root & .042 & $2.165^{\mathrm{c}}$ & 9.000 & 464.000 & .023 & .040 \\
\hline \multirow{4}{*}{$\begin{array}{l}\text { Promotion * } \\
\text { Design of } \\
\text { Packaging }\end{array}$} & Pillai's Trace & .042 & 3.345 & 6.000 & 928.000 & .003 & .021 \\
\hline & Wilks' Lambda & .958 & $3.357^{\mathrm{b}}$ & 6.000 & 926.000 & .003 & .021 \\
\hline & Hotelling's Trace & .044 & 3.369 & 6.000 & 924.000 & .003 & .021 \\
\hline & Roy's Largest Root & .038 & $5.837^{\mathrm{c}}$ & 3.000 & 464.000 & .001 & .036 \\
\hline \multirow{4}{*}{$\begin{array}{l}\text { Nutrient } \\
\text { Content } * \\
\text { Design of } \\
\text { Packaging }\end{array}$} & Pillai's Trace & .039 & 4.578 & 4.000 & 928.000 & .001 & .019 \\
\hline & Wilks' Lambda & .961 & $4.612^{b}$ & 4.000 & 926.000 & .001 & .020 \\
\hline & Hotelling's Trace & .040 & 4.646 & 4.000 & 924.000 & .001 & .020 \\
\hline & Roy's Largest Root & .040 & $9.226^{\mathrm{c}}$ & 2.000 & 464.000 & .000 & .038 \\
\hline \multirow{4}{*}{$\begin{array}{l}\text { Price * } \\
\text { Promotion * } \\
\text { Nutrient } \\
\text { Content }\end{array}$} & Pillai's Trace & .007 & .870 & 4.000 & 928.000 & .482 & .004 \\
\hline & Wilks' Lambda & .993 & $.869^{\mathrm{b}}$ & 4.000 & 926.000 & .482 & .004 \\
\hline & Hotelling's Trace & .008 & .868 & 4.000 & 924.000 & .483 & .004 \\
\hline & Roy's Largest Root & .007 & $1.558^{\mathrm{c}}$ & 2.000 & 464.000 & .212 & .007 \\
\hline \multirow{4}{*}{$\begin{array}{l}\text { Price * } \\
\text { Promotion * } \\
\text { Design of } \\
\text { Packaging }\end{array}$} & Pillai's Trace & .000 &. $\mathrm{~b}$ & .000 & .000 & . & . \\
\hline & Wilks' Lambda & 1.000 & b & .000 & 463.500 & . & . \\
\hline & \begin{tabular}{|l|} 
Hotelling's Trace \\
\end{tabular} & .000 &. $\mathrm{~b}$ & .000 & 2.000 & . & . \\
\hline & Roy's Largest Root & .000 & $.000^{\mathrm{b}}$ & 2.000 & 462.000 & 1.000 & .000 \\
\hline \multirow{4}{*}{$\begin{array}{l}\text { Price * Nutrient } \\
\text { Content } * \\
\text { Design of } \\
\text { Packaging }\end{array}$} & Pillai's Trace & .000 & $.036^{\mathrm{b}}$ & 2.000 & 463.000 & .965 & .000 \\
\hline & \begin{tabular}{|l|} 
Wilks' Lambda \\
\end{tabular} & 1.000 & $.036^{\mathrm{b}}$ & 2.000 & 463.000 & .965 & .000 \\
\hline & \begin{tabular}{|l} 
Hotelling's Trace \\
\end{tabular} & .000 & $.036^{\mathrm{b}}$ & 2.000 & 463.000 & .965 & .000 \\
\hline & \begin{tabular}{|l} 
Roy's Largest Root \\
\end{tabular} & .000 & $.036^{\mathrm{b}}$ & 2.000 & 463.000 & .965 & .000 \\
\hline \multirow{4}{*}{$\begin{array}{l}\text { Promotion * } \\
\text { Nutrient } \\
\text { Content * } \\
\text { Design of } \\
\text { Packaging }\end{array}$} & Pillai's Trace & .012 & .548 & 10.000 & 928.000 & .857 & .006 \\
\hline & Wilks' Lambda & .988 & $.548^{b}$ & 10.000 & 926.000 & .856 & .006 \\
\hline & Hotelling's Trace & .012 & .549 & 10.000 & 924.000 & .856 & .006 \\
\hline & Roy's Largest Root & .012 & $1.093^{\mathrm{c}}$ & 5.000 & 464.000 & .363 & .012 \\
\hline \multirow{4}{*}{$\begin{array}{l}\text { Price * } \\
\text { Promotion * } \\
\text { Nutrient } \\
\text { Content * } \\
\text { Design of } \\
\text { Packaging } \\
\end{array}$} & Pillai's Trace & .000 &. & .000 & .000 & . & $\cdot$ \\
\hline & \begin{tabular}{|l|} 
Wilks' Lambda \\
\end{tabular} & 1.000 &. & .000 & 463.500 & . & . \\
\hline & Hotelling's Trace & .000 &. & .000 & 2.000 & . & . \\
\hline & Roy's Largest Root & .000 & $.000^{\mathrm{b}}$ & 2.000 & 462.000 & 1.000 & .000 \\
\hline
\end{tabular}


The statistic analysis is an upper bound on $\mathrm{F}$ that yields a lower bound on the significance level. Multivariate significance test showed fifteen different centroids based on tests of Pillai's Trade, Wilks Lambda, Hotteling's Trace and Roy's Largest Root. Based on F-test with 95 per cent level of significance, the result of the four factors of price, promotion, nutrient content and design of packaging was not equal to zero, and there was a difference between the four marketing programs of fertilizer products. The result of significance of F-test using the four test on multivariate analysis revealed significant test results on price, promotion, nutrient content, design of packaging, interaction between price and design of packaging, interaction between promotion and nutrient content, interaction between promotion and design of packaging, and interaction between nutrient content and design of packaging. In MANOVA Test with more than one independent variable, there were fifteen main effect tests with four independent variables consisting of price, promotion, nutrient content, the design of packaging and eleven interaction tests among the four independent variables. It shows that price, promotion, nutrient content and design of packaging influence farmer preference for marketing program used both by local and imported fertilizers.

Moreover, price, promotion, nutrient content and design of packaging simultaneously influence farmer attitude to marketing program of fertilizer product. Partial Eta Squared (PES) based on roy's largest root, show a promotion factor has the highest total variance with $5.2 \%$ of the total variance and design of packaging factor has the lowest factor with $1.7 \%$ of the total variance. The multivariate test has a powerful influence on rejecting the null hypothesis of the study and gives a conclusion on the existence on an influence of price, promotion, nutrient content and design of packaging on farmer marketing preference for fertilizer product they use for their horticultural business farm.

\section{Significance Test of Variance Error}

Variance test for error is shown by Levene's test. The test shows significance result that indicates a non-equal error on variance farmer preference for local fertilizer as well as farmer preference for imported fertilizer.

TABLE V

LEVENE'S TEST OF EQUALITY OF ERROR VARIANCES ${ }^{A}$

\begin{tabular}{|l|c|c|c|c|}
\hline & F & df1 & df2 & Sig. \\
\hline $\begin{array}{l}\text { Farmer } \\
\text { Preference for } \\
\text { Local Fertilizer }\end{array}$ & 2.793 & 48 & 464 & .000 \\
\hline $\begin{array}{l}\text { Farmer } \\
\begin{array}{l}\text { Preference for } \\
\text { Imported } \\
\text { Fertilizer }\end{array}\end{array}$ & 2.734 & 48 & 464 & .000 \\
\hline
\end{tabular}

\section{Univariate Significant Test}

F-test statistic is used to represent significant univariate test. The study showed that by $\alpha=5 \%$, the only main effect of price, promotion, nutrient content, and promotion and design of packaging were significant. Others factors i.e. design of packaging were not significant whether for an individual factor of a design of packaging and interaction simultaneous among factors. It can be interpreted that factors of price, promotion and design of packaging influence both farmer attitude to local fertilizers and imported fertilizers and only promotion and design of packaging influence both farmer preference for local fertilizers and imported fertilizers. The highest score for Partial Eta Squared was for promotion of farmer preference for local fertilizer (0.046), while other cases showed the lower score.

TABLE VI

TESTS OF BETWEEN-SUBJECTS EFFECTS

\begin{tabular}{|c|c|c|c|c|c|c|c|}
\hline Source & Dependent Variable & $\begin{array}{c}\text { Type III Sum } \\
\text { of Squares }\end{array}$ & df & Mean Square & $\mathrm{F}$ & Sig. & $\begin{array}{l}\text { Partial Eta } \\
\text { Squared }\end{array}$ \\
\hline \multirow[t]{2}{*}{ Corrected Model } & $\begin{array}{l}\text { Farmer Attitude to Local } \\
\text { Fertilizer }\end{array}$ & $3726.793^{\mathrm{a}}$ & 48 & 77.642 & 4.095 & .000 & .298 \\
\hline & $\begin{array}{l}\text { Farmer Attitude to Imported } \\
\text { Fertilizer }\end{array}$ & $3574.346^{\mathrm{b}}$ & 48 & 74.466 & 3.363 & .000 & .258 \\
\hline \multirow[t]{2}{*}{ Intercept } & $\begin{array}{l}\text { Farmer Attitude to Local } \\
\text { Fertilizer }\end{array}$ & 23896.809 & 1 & 23896.809 & 1260.454 & .000 & .731 \\
\hline & $\begin{array}{l}\text { Farmer Attitude to Imported } \\
\text { Fertilizer }\end{array}$ & 28998.013 & 1 & 28998.013 & 1309.581 & .000 & .738 \\
\hline \multirow[t]{2}{*}{ Price } & $\begin{array}{l}\text { Farmer Attitude to Local } \\
\text { Fertilizer }\end{array}$ & 300.480 & 4 & 75.120 & 3.962 & .004 & .033 \\
\hline & $\begin{array}{l}\text { Farmer Attitude to Imported } \\
\text { Fertilizer }\end{array}$ & 300.574 & 4 & 75.143 & 3.394 & .009 & .028 \\
\hline \multirow[t]{2}{*}{ Promotion } & $\begin{array}{l}\text { Farmer Attitude to Local } \\
\text { Fertilizer }\end{array}$ & 428.685 & 3 & 142.895 & 7.537 & .000 & .046 \\
\hline & $\begin{array}{l}\text { Farmer Attitude to Imported } \\
\text { Fertilizer }\end{array}$ & 303.511 & 3 & 101.170 & 4.569 & .004 & .029 \\
\hline \multirow[t]{2}{*}{ Nutrient Content } & $\begin{array}{l}\text { Farmer Attitude to Local } \\
\text { Fertilizer }\end{array}$ & 376.690 & 3 & 125.563 & 6.623 & .000 & .041 \\
\hline & $\begin{array}{l}\text { Farmer Attitude to Imported } \\
\text { Fertilizer }\end{array}$ & 428.230 & 3 & 142.743 & 6.446 & .000 & .040 \\
\hline $\begin{array}{l}\text { Design of } \\
\text { Packaging }\end{array}$ & $\begin{array}{l}\text { Farmer Attitude to Local } \\
\text { Fertilizer }\end{array}$ & 30.218 & 1 & 30.218 & 1.594 & .207 & .003 \\
\hline
\end{tabular}




\begin{tabular}{|c|c|c|c|c|c|c|c|}
\hline Source & Dependent Variable & $\begin{array}{l}\text { Type III Sum } \\
\text { of Squares }\end{array}$ & $\mathrm{df}$ & Mean Square & $\mathrm{F}$ & Sig. & $\begin{array}{l}\text { Partial Eta } \\
\text { Squared }\end{array}$ \\
\hline & $\begin{array}{l}\text { Farmer Attitude to Imported } \\
\text { Fertilizer }\end{array}$ & .383 & 1 & .383 & .017 & .895 & .000 \\
\hline \multirow[t]{2}{*}{$\begin{array}{l}\text { Price } * \\
\text { Promotion }\end{array}$} & $\begin{array}{l}\text { Farmer Attitude to Local } \\
\text { Fertilizer }\end{array}$ & 58.943 & 5 & 11.789 & .622 & .683 & .007 \\
\hline & $\begin{array}{l}\text { Farmer Attitude to Imported } \\
\text { Fertilizer }\end{array}$ & 46.428 & 5 & 9.286 & .419 & .835 & .004 \\
\hline \multirow[t]{2}{*}{$\begin{array}{l}\text { Price } * \text { Nutrient } \\
\text { Content }\end{array}$} & $\begin{array}{l}\text { Farmer Attitude to Local } \\
\text { Fertilizer }\end{array}$ & 73.550 & 4 & 18.388 & .970 & .424 & .008 \\
\hline & $\begin{array}{l}\text { Farmer Attitude to Imported } \\
\text { Fertilizer }\end{array}$ & 68.270 & 4 & 17.067 & .771 & .545 & .007 \\
\hline \multirow{2}{*}{$\begin{array}{l}\text { Price } * \\
\text { Design of } \\
\text { Packaging }\end{array}$} & $\begin{array}{l}\text { Farmer Attitude to Local } \\
\text { Fertilizer }\end{array}$ & 130.250 & 2 & 65.125 & 3.435 & .033 & .015 \\
\hline & $\begin{array}{l}\text { Farmer Attitude to Imported } \\
\text { Fertilizer }\end{array}$ & 99.890 & 2 & 49.945 & 2.256 & .106 & .010 \\
\hline \multirow[t]{2}{*}{$\begin{array}{l}\text { Promotion } * \\
\text { Nutrient Content }\end{array}$} & $\begin{array}{l}\text { Farmer Attitude to Local } \\
\text { Fertilizer }\end{array}$ & 340.188 & 9 & 37.799 & 1.994 & .038 & .037 \\
\hline & $\begin{array}{l}\text { Farmer Attitude to Imported } \\
\text { Fertilizer }\end{array}$ & 331.144 & 9 & 36.794 & 1.662 & .096 & .031 \\
\hline \multirow{2}{*}{$\begin{array}{l}\text { Promotion } * \\
\text { Design of } \\
\text { Packaging }\end{array}$} & $\begin{array}{l}\text { Farmer Attitude to Local } \\
\text { Fertilizer }\end{array}$ & 290.219 & 3 & 96.740 & 5.103 & .002 & .032 \\
\hline & $\begin{array}{l}\text { Farmer Attitude to Imported } \\
\text { Fertilizer }\end{array}$ & 213.993 & 3 & 71.331 & 3.221 & .023 & .020 \\
\hline \multirow{2}{*}{$\begin{array}{l}\text { Nutrient Content } \\
* \\
\text { Design of } \\
\text { Packaging }\end{array}$} & $\begin{array}{l}\text { Farmer Attitude to Local } \\
\text { Fertilizer }\end{array}$ & 203.449 & 2 & 101.724 & 5.366 & .005 & .023 \\
\hline & $\begin{array}{l}\text { Farmer Attitude to Imported } \\
\text { Fertilizer }\end{array}$ & 78.340 & 2 & 39.170 & 1.769 & .172 & .008 \\
\hline \multirow{2}{*}{$\begin{array}{l}\text { Price * } \\
\text { Promotion * } \\
\text { Nutrient Content }\end{array}$} & $\begin{array}{l}\text { Farmer Attitude to Local } \\
\text { Fertilizer }\end{array}$ & 57.118 & 2 & 28.559 & 1.506 & .223 & .006 \\
\hline & $\begin{array}{l}\text { Farmer Attitude to Imported } \\
\text { Fertilizer }\end{array}$ & 48.652 & 2 & 24.326 & 1.099 & .334 & .005 \\
\hline \multirow{2}{*}{$\begin{array}{l}\text { Price } * \\
\text { Promotion } * \\
\text { Design of } \\
\text { Packaging }\end{array}$} & $\begin{array}{l}\text { Farmer Attitude to Local } \\
\text { Fertilizer }\end{array}$ & .000 & 0 & . & . & . & .000 \\
\hline & $\begin{array}{l}\text { Farmer Attitude to Imported } \\
\text { Fertilizer }\end{array}$ & .000 & 0 & . & . & . & .000 \\
\hline \multirow{2}{*}{$\begin{array}{l}\text { Price } * \text { Nutrient } \\
\text { Content } * \text { Design } \\
\text { of Packaging }\end{array}$} & $\begin{array}{l}\text { Farmer Attitude to Local } \\
\text { Fertilizer }\end{array}$ & .786 & 1 & .786 & .041 & .839 & .000 \\
\hline & $\begin{array}{l}\text { Farmer Attitude to Imported } \\
\text { Fertilizer }\end{array}$ & .295 & 1 & .295 & .013 & .908 & .000 \\
\hline \multirow{2}{*}{$\begin{array}{l}\text { Promotion } * \\
\text { Nutrient Content } \\
* \text { Design of } \\
\text { Packaging }\end{array}$} & $\begin{array}{l}\text { Farmer Attitude to Local } \\
\text { Fertilizer }\end{array}$ & 37.619 & 5 & 7.524 & .397 & .851 & .004 \\
\hline & $\begin{array}{l}\text { Farmer Attitude to Imported } \\
\text { Fertilizer }\end{array}$ & 6.670 & 5 & 1.334 & .060 & .998 & .001 \\
\hline \multirow{2}{*}{$\begin{array}{l}\text { Price * } \\
\text { Promotion* } \\
\text { Nutrient Content } \\
* \text { Design of } \\
\text { Packaging }\end{array}$} & $\begin{array}{l}\text { Farmer Attitude to Local } \\
\text { Fertilizer }\end{array}$ & .000 & 0 & . & . & . & .000 \\
\hline & $\begin{array}{l}\text { Farmer Attitude to Imported } \\
\text { Fertilizer }\end{array}$ & .000 & 0 & . & . & . & .000 \\
\hline \multirow[t]{2}{*}{ Error } & $\begin{array}{l}\text { Farmer Attitude to Local } \\
\text { Fertilizer }\end{array}$ & 8796.926 & 464 & 18.959 & & & \\
\hline & $\begin{array}{l}\text { Farmer Attitude to Imported } \\
\text { Fertilizer }\end{array}$ & 10274.336 & 464 & 22.143 & & & \\
\hline \multirow[t]{2}{*}{ Total } & $\begin{array}{l}\text { Farmer Attitude to Local } \\
\text { Fertilizer }\end{array}$ & 494236.000 & 513 & & & & \\
\hline & $\begin{array}{l}\text { Farmer Attitude to Imported } \\
\text { Fertilizer }\end{array}$ & 608646.000 & 513 & & & & \\
\hline \multirow[t]{2}{*}{ Corrected Total } & $\begin{array}{l}\text { Farmer Attitude to Local } \\
\text { Fertilizer }\end{array}$ & 12523.719 & 512 & & & & \\
\hline & $\begin{array}{l}\text { Farmer Attitude to Imported } \\
\text { Fertilizer }\end{array}$ & 13848.682 & 512 & & & & \\
\hline
\end{tabular}


TABLE VII

PRICE FACTOR

\begin{tabular}{|c|c|c|c|c|c|}
\hline \multirow{2}{*}{ Dependent Variable } & \multirow{2}{*}{ Price per $\mathrm{Kg}$} & \multirow{2}{*}{ Mean } & \multirow{2}{*}{ Std. Error } & \multicolumn{2}{|c|}{$95 \%$ Confidence Interval } \\
\hline & & & & Lower Bound & Upper Bound \\
\hline \multirow{5}{*}{$\begin{array}{l}\text { Farmer Attitude to Local } \\
\text { Fertilizer }\end{array}$} & IDR 4,000 - IDR 9,000 & $31.042^{\mathrm{a}}$ & .516 & 30.027 & 32.056 \\
\hline & IDR 9,000 - IDR 11,000 & $28.164^{\mathrm{a}}$ & .890 & 26.415 & 29.913 \\
\hline & IDR 11,000 - IDR 15,000 & $31.600^{\mathrm{a}}$ & 1.742 & 28.177 & 35.023 \\
\hline & IDR 16,000 - IDR 20,000 & $33.000^{\mathrm{a}}$ & 4.354 & 24.444 & 41.556 \\
\hline & >IDR 20,000 & $23.833^{\mathrm{a}}$ & 2.295 & 19.324 & 28.343 \\
\hline \multirow{5}{*}{$\begin{array}{l}\text { Farmer Attitude to Imported } \\
\text { Fertilizer }\end{array}$} & IDR 4,000 - IDR 9,000 & $33.712^{\mathrm{a}}$ & .558 & 32.615 & 34.808 \\
\hline & IDR 9,000 - IDR 11,000 & $30.968^{\mathrm{a}}$ & .962 & 29.078 & 32.859 \\
\hline & IDR 11,000 - IDR 15,000 & $35.400^{\mathrm{a}}$ & 1.882 & 31.701 & 39.099 \\
\hline & IDR 16,000 - IDR 20,000 & $37.000^{\mathrm{a}}$ & 4.706 & 27.753 & 46.247 \\
\hline & >IDR 20,000 & $26.833^{\mathrm{a}}$ & 2.480 & 21.960 & 31.707 \\
\hline
\end{tabular}

The results of Estimated Marginal Means show the details of the influence of each factor. In the case of price factor, the price ranging between IDR 16,000 to IDR 20,000 per $\mathrm{kg}$ showed the highest influence both on farmer preference for local fertilizer $($ mean $=33.000)$ and farmer preference for imported fertilizer $($ mean $=37.000)$, compared to the influence of other price range both on farmer attitude to local fertilizers and imported fertilizers. It implies that fertilizer products with a price range of IDR 16,000 to IDR 20,000 per $\mathrm{kg}$ will possibly give more significant change on how farmers as fertilizer product customers believe on product and compare it to the fertilizer product performance.

TABLE VIII

PROMOTION FACTORS

\begin{tabular}{|l|l|l|l|l|c|}
\hline \multirow{2}{*}{ Dependent Variable } & \multicolumn{1}{|c|}{ Promotion } & \multirow{2}{*}{ Mean } & \multirow{2}{*}{ Std. Error } & \multicolumn{2}{c|}{ 95\% Confidence Interval } \\
\cline { 3 - 6 } & & & \multicolumn{2}{|c|}{ Lower Bound } & Upper Bound \\
\hline \multirow{3}{*}{$\begin{array}{l}\text { Farmer Preference for Local } \\
\text { Fertilizer }\end{array}$} & Farm Demonstration Plot & $31.778^{\mathrm{a}}$ & .683 & 30.436 & 33.120 \\
\cline { 2 - 6 } & Farmer Training & $31.967^{\mathrm{a}}$ & 1.069 & 29.866 & 34.068 \\
\cline { 2 - 6 } & Farmer Assistance & $26.275^{\mathrm{a}}$ & .889 & 24.528 & 28.022 \\
\cline { 2 - 6 } & Free Product Sample & $29.476^{\mathrm{a}}$ & 1.041 & 27.430 & 31.522 \\
\hline \multirow{3}{*}{$\begin{array}{l}\text { Farmer Preference for } \\
\text { Imported Fertilizer }\end{array}$} & Farm Demostration Plot & $34.390^{\mathrm{a}}$ & .738 & 32.940 & 35.840 \\
\cline { 2 - 6 } & Farmer Training & $34.396^{\mathrm{a}}$ & 1.155 & 32.125 & 36.666 \\
\cline { 2 - 6 } & Farmer Assistance & $29.429^{\mathrm{a}}$ & .961 & 27.541 & 31.317 \\
\cline { 2 - 6 } & Free Product Sample & $32.904^{\mathrm{a}}$ & 1.125 & 30.692 & 35.115 \\
\hline
\end{tabular}

Estimated Marginal Means related to the second factor promotion revealed promotion by giving training to the farmers is the most influential way of fertilizer product promotion on both farmer preference for local fertilizers (mean $=31.967)$ and farmer preference for imported fertilizers $($ mean $=34.396)$. However, the promotional medium with farm demonstration plot slightly showed the same level of influence on farmer preference for local fertilizers and imported fertilizers. In other words, other promotional media such as farmer assistance and free product samples give lower influence on farmer preference both for local and imported fertilizers. Farmers will possibly have higher trust in fertilizer product performance through a promotional media of farmer training and farm demonstration plot.

TABLE IX

NUTRIENT CONTENT FACTORS

\begin{tabular}{|c|c|c|c|c|c|}
\hline \multirow[b]{2}{*}{ Dependent Variable } & \multirow[b]{2}{*}{ Nutrient Content } & \multirow[b]{2}{*}{ Mean } & \multirow[b]{2}{*}{ Std. Error } & \multicolumn{2}{|c|}{ 95\% Confidence Interval } \\
\hline & & & & Lower Bound & Upper Bound \\
\hline \multirow[t]{4}{*}{$\begin{array}{l}\text { Farmer Preference for } \\
\text { Local Fertilizer }\end{array}$} & $\begin{array}{l}\text { Local fertilizer nutrient with } \\
\text { addition of micro plant nutrient } \\
\text { and plant vitamin }\end{array}$ & $28.744^{\mathrm{a}}$ & .942 & 26.893 & 30.595 \\
\hline & Local fertilizer plant nutrient & $27.778^{\mathrm{a}}$ & .977 & 25.858 & 29.698 \\
\hline & $\begin{array}{l}\text { Imported fertilizer nutrient with } \\
\text { addition of micro plant nutrient } \\
\text { and plant vitamin }\end{array}$ & $31.973^{\mathrm{a}}$ & .698 & 30.601 & 33.345 \\
\hline & Imported fertilizer plant nutrient & $29.032^{\mathrm{a}}$ & .867 & 27.329 & 30.735 \\
\hline $\begin{array}{l}\text { Farmer Preference for } \\
\text { Imported Fertilizer }\end{array}$ & $\begin{array}{l}\text { Local fertilizer nutrient content } \\
\text { with addition of micro plant } \\
\text { nutrient and plant vitamin }\end{array}$ & $31.645^{\mathrm{a}}$ & 1.018 & 29.644 & 33.646 \\
\hline
\end{tabular}




\begin{tabular}{|c|c|c|c|c|c|}
\hline \multirow{5}{*}{ Dependent Variable } & \multirow[b]{2}{*}{ Nutrient Content } & \multirow[b]{2}{*}{ Mean } & \multirow[b]{2}{*}{ Std. Error } & \multicolumn{2}{|c|}{ 95\% Confidence Interval } \\
\hline & & & & Lower Bound & Upper Bound \\
\hline & Local fertilizer plant nutrient & $30.611^{\mathrm{a}}$ & 1.056 & 28.536 & 32.686 \\
\hline & $\begin{array}{l}\text { Imported fertilizer nutrient with } \\
\text { addition of micro plant nutrient } \\
\text { and plant vitamin }\end{array}$ & $34.458^{\mathrm{a}}$ & .755 & 32.975 & 35.941 \\
\hline & Imported fertilizer plant nutrient & $32.374^{\mathrm{a}}$ & .936 & 30.533 & 34.214 \\
\hline
\end{tabular}

The third factor, namely farmer preference for fertilizer nutrient content, shows that imported fertilizers with addition of plant micronutrient plant vitamin give higher influence on farmer marketing attitude to both local fertilizers $($ mean $=31.973$ ) and imported fertilizers (mean
34.458). This shows that fertilizer products with nutrient content in imported fertilizer products give a higher contribution to farmer marketing behavior related to farmer assessment to buy both local or imported fertilizer.

TABLE X

DESIGN OF PACKAGING FACTOR

\begin{tabular}{|l|l|l|l|l|c|}
\hline \multicolumn{1}{|c|}{ Dependent Variable } & \multirow{2}{*}{ Design of Packaging } & \multirow{2}{*}{ Std. Error } & \multicolumn{2}{|c|}{$95 \%$ Confidence Interval } \\
\cline { 3 - 6 } $\begin{array}{l}\text { Farmer Preference for Local } \\
\text { Fertilizer }\end{array}$ & $\begin{array}{l}\text { Packaging with additional image } \\
\text { design }\end{array}$ & $29.823^{\mathrm{a}}$ & .463 & 28.914 & 30.732 \\
\cline { 2 - 6 } & $\begin{array}{l}\text { Packaging without additional } \\
\text { image design }\end{array}$ & $30.142^{\mathrm{a}}$ & .932 & 28.310 & 31.974 \\
\hline $\begin{array}{l}\text { Farmer Preference for } \\
\text { Imported Fertilizer }\end{array}$ & $\begin{array}{l}\text { Packaging with additional image } \\
\text { design }\end{array}$ & $33.314^{\mathrm{a}}$ & .500 & 32.332 & 34.297 \\
\cline { 2 - 7 } & $\begin{array}{l}\text { Packaging without additional } \\
\text { image design }\end{array}$ & $31.838^{\mathrm{a}}$ & 1.008 & 29.858 & 33.818 \\
\hline
\end{tabular}

The last factor, namely farmer preference for the design of packaging, shows that fertilizer product packaging without additional image tends to give a higher contribution on farmer preference for local fertilizers (mean $=30.142$ ). Meanwhile, farmer preference for imported fertilizer shows a different result, as preference for fertilizer product packaging with additional image design gives a higher contribution on farmer preference for choosing imported fertilizer $($ mean $=33.314)$. These mean that what attracts customer in fertilizer products related to product attributes in the design of packaging is distinct between local and imported fertilizers. Local fertilizer products without additional image design can change farmer-marketing behavior more easily on choosing local fertilizers, while on the contrary imported fertilizers with additional design on packaging tend to easily move farmers' attitude and effect on imported fertilizer product choice.

\section{F. Discussion}

Marketing strategy becomes a key factor to improve the decision related to market segment and market targets. It is used to determine recent situation to find a new market space in the global competition. This study describes that four observed product attributes on farmer preferences for marketing program of fertilizer could influence the fertilizer marketplace.

Firstly, the price has taken a role as an important factor in global competition. The price has a direct influence on consumer perception of buying a fertilizer [19]. Price becomes a significant attribute to increase marketplace and becomes a sensitive factor in consumer preference for local and imported fertilizers. Fertilizer price range of IDR 16,000 to IDR 20,000 per $\mathrm{kg}$ is the farmer preference for both local and imported ones. The farmer does not prefer to buy the lowest price, but they see the price to represent fertilizer quality. The farmer does not buy the cheapest fertilizer price, therefore, the strategy is not to set the lowest price among the competition [5]. In addition, customer is likely willing to buy a premium price sustainable fertilizer [20].

Secondly, fertilizer promotion would be highly effective if industries conduct farm demonstration plot for local and imported fertilizers. In addition, promotion through farmer training would be effective. The farmer prefers to be approached through training and demonstration plot. Farmer agricultural input adoption can be done through participatory and training extension system [21].

Thirdly, the nutrient contained in the local and imported fertilizer is significantly important along with additional micro-nutrient content and plant vitamin. Fertilizer industries (specifically local fertilizer industry) should increase the nutrient content of fertilizer products as a marketing strategy to grasp competitiveness and robust market strategy[12].

Fourthly, the design of the packaging is the only one factor that has differences between local and imported fertilizers. The farmer prefers local fertilizer product packaging without an additional image. Information such as text from the label and brand on the packaging has fulfilled farmer information searching. On the other hand, the farmer prefers imported fertilizer product packaging with an additional image. The design of the packaging is playing a role in the marketing strategy to self-appeal, providing product information, brand image and awareness [22]. Therefore, the producer should provide the packaging appearance that adjusted with consumer preference. 


\section{CONCLUSION}

The result of this study gives some insight about horticultural farmers preference for fertilizers in Java island, Indonesia. It can represent how the behavior of small-scale farmers in Asia reacts to the local and imported fertilizers. There is distinction of farmer preferences for local and imported fertilizers in terms of design packaging. However, in terms of price, promotion, and nutrition, the farmer preference is similar to both fertilizer sources. This finding serves as a reference to companies in improving their strategy to win the competition. Local fertilizer companies should provide fertilizer with a price range of IDR 16,000 to IDR 20,000 per $\mathrm{kg}$, promoting through farm demonstration plot, addition of micro-nutrient content and plant vitamin, and simple design without images.

Future research should focus on preference at a bigger scale of the farmers because this research only represents the behavior on the small-scale farmers spread over Java Island. The research result might be different if it apply in a largescale farmer (such as a commercial farming or a company) because this type of farmer has different characteristic with small-scale farmer which will influence to their preference of fertilizer.

\section{ACKNOWLEDGMENT}

The authors gratefully acknowledge Universitas Padjadjaran for research funding.

\section{REFERENCES}

[1] J. A. Dixon, D. P. Gibbon, and A. Gulliver. Farming systems and poverty: improving farmers' livelihoods in a changing world. Food \& Agriculture Org, 2001.

[2] S. P. Mantel and F. R. Kardes. The Role of Direction of Comparison, Attribute-Based Processing, and Attitude-Based Processing in Consumer Preference. Journal of Consumer Research, 25 (4), 335352, 1999.

[3] D. R. Lehmann and J. O'Shaughnessy. Difference in Attribute Importance for Different Industrial Products. Journal of Marketing, 38 (2), 36-42, 1974.

[4] G. A. Knight. Consumer Preferences for Foreign and Domestic Products. Journal of Consumer Marketing, 16 (2), 151-162, 1999.

[5] T. F. Funk. Fertilizer Buying Behavior of Ontario Farmers. Canadian Journal of Agricultural Economics/Revue Canadienne D'agroeconomie, 30(3), 319-332, 1982.

[6] R. Lotriet, C. Bisschoff and A. Kole. Assessing Fertilizer Buying Behavior Of Emerging Farmers in A South African Grain Producing Area. Problems and Perspectives in Management, 15(2-2), 456-467, 2017.
[7] K. Goulding, S. Jarvis, and A.Whitmore. Optimizing Nutrient Management for Farm Systems. Philosophical Transactions of the Royal Society B: Biological Sciences, 363(149), 667-680, 2008.

[8] J. Herche. A Note On The Predictive Validity of The CETSCALE. Journal of The Academy of Marketing Science. 20, Summer, 261264, 1992.

[9] K. L. Keller. Conceptualizing, Measuring, and Managing CustomerBased Brand Equity. The Journal of Marketing : 1-22, 193.

[10] D. Gemina , T. T. Andari , and I. C. Kusuma. Consumer Behavior on The Choice of Typical Regional Food Products Based on External and Internal Factors, Perception, Attitude and Consumer Preference. International Journal on Advanced Science Engineering Information Technology. 3 (2), 43-46, 2013

[11] T. Perdana, E. Renaldy, H. N. Utami, A. H. Sadeli, M. A. Heryanto, T. Ginanjar, A. Sesy, F. R. Hermiatin and S. Sanjaya. Farmers Behavior on Using Fertilizer in West Java. AIP Conference Proceedings. Vol. 1927. No. 1. AIP Publishing, 2018.

[12] H. N. Utami, A. H. Sadeli, T. Perdana, E. Renaldy, M. A. Heryanto, A. Sesy, F. R. Hermiatin, T. Ginanjar, and S. Sanjaya. Consumer Preference of Fertilizer in West Java Using Multi-Dimensional Scaling Approach. AIP Conference Proceedings. Vol. 1927. No. 1. AIP Publishing, 2018.

[13] J. Dahlin, V. Halbherr, P. Kurz, M. Nelles and C. Herbes. Marketing Green Fertilizers: Insights into Consumer Preferences. Sustainability, 8 (11), 2-15, 2016.

[14] H. N. Utami, A. H. Sadeli and T. Perdana. Customer Value Creation of Fresh Tomatoes through Branding and Packaging as Customer Perceived Quality . J. ISSAAS. 22(1), 123-136, 2016.

[15] P. Silayoi and M. Speece. The Importance of Packaging Attributes: A Conjoint Analysis Approach. 41 (11/12), 1495-1517, 2007.

[16] Asosiasi Pedagang Pupuk Indonesia. statistic APPI. Accessed through: http://www.appi.or.id/?statistic. Access date: 01/03/2018.

[17] M. Hossain and V. P. Singh. Fertilizer use in Asian agriculture: implications for sustaining food security and the environment. Nutrient Cycling in Agroecosystems, 57(2), 155-169, 2000.

[18] C. J. Huberty. and S. Olejnik. Applied MANOVA and Discriminant Analysis Second- Edition. A John Willey and Son Inc.: New Jersey, 2006.

[19] K. B. Monroe. Buyers' Subjective Perceptions of Price. Journal of marketing research: 70-80, 1973.

[20] H. Khatchatryan, D. H. Suh, G. Zhou and M. Dukes. Sustainable Urban Landscaping: Consumer Preferences and Willingness to Pay for Turfgrass Fertilizers. Canadian Journal of Agricultural Economics, 65(3), 1-23, 2016.

[21] K. Belay. Agricultural Extension in Ethiopia: The Case of Participatory Demonstration and Training Extension System. Journal of Social Development in Africa. 18 (1), 49-84, 2003.

[22] S. O. Sarpong. Exploring the concept of standout in structural packaging-challenges and prospects in Ghana. Diss, 2015. 